\title{
Peroneal nerve decompression
}

\author{
Mark Corriveau, MD, Jacob D. Lescher, BS, and Amgad S. Hanna, MD
}

Department of Neurological Surgery, University of Wisconsin School of Medicine and Public Health, Madison, Wisconsin

Peroneal neuropathy is a common pathology encountered by neurosurgeons. Symptoms include pain, numbness, and foot drop. When secondary to compression of the nerve at the fibular head, peroneal (fibular) nerve release is a low-risk procedure that can provide excellent results with pain relief and return of function. In this video, the authors highlight key operative techniques to ensure adequate decompression of the nerve while protecting the 3 major branches, including the superficial peroneal nerve, deep peroneal nerve, and recurrent genicular (articular) branches. Key steps include positioning, circumferential nerve dissection, fascial opening, isolation of the major branches, and closure.

The video can be found here: https://youtu.be/0y9oE8w1FIU.

KEYWORDS common peroneal nerve; deep peroneal nerve; fibular nerve; recurrent genicular branch; superficial peroneal nerve; video 